\title{
Serum albumin molecular mobility in water solutions, containing iron chloride III
}

\author{
Victoria V. Gibizova*, Viktor A. Sapozhnikov, Ksenia V.Fedorova, and Galina P. Petrova \\ Department of Physics, M.V. Lomonosov Moscow State University, Leninskiye Gory 1, building 2, Moscow GSP-1, \\ 119991, Russian Federation \\ *e-mail: gibizova@physics.msu.ru
}

\begin{abstract}
The lack or overload of iron in living organism can cause different diseases, including those related to the serum blood. In this connection the basic blood proteins, in particular, albumin, can change their charge, conformation, molecular mobility, etc. Due to this fact, it is of undoubtful interest to study the molecular dynamical processes that occur in water solutions of serum albumin under the effect of iron ions. In the present work we performed comparative studies of optical properties of bovine serum albumin (BSA) water solutions and BSA solutions with the addition of Iron(III) chloride by light scattering. (C) 2016 Journal of Biomedical Photonics \& Engineering.
\end{abstract}

Keywords: dynamic light scattering, static light scattering, translation diffusion coefficient, hydrodynamic radius, intermolecular interaction coefficient

Paper \#3128 received 2016.11.20; accepted for publication 2016.12.29; published online 2016.12.31. doi: 10.18287/JBPE16.02.040304. [Saratov Fall Meeting 2016 Special Issue].

\section{References}

1. P. T. Lieu, M. Heiskala, P. A. Peterson, and Y. Yang, "The roles of iron in health and disease," Molecular Aspects of Medicine 22(1-2), 1-87 (2001).

2. Z. Shu-hong, F. Yong-shan, F. Shuo, and Z. Yun-feng, "Microdetermination of proteins by resonance light scattering technique based on aggregation of ferric nanoparticles," Spectrochimica Acta Part A 72, 748-752 (2008).

3. B. D. Fair, and A. M. Jamieson, "Effect of Electrodynamic Interactions on the Translational Diffusion of Bovine Serum Albumin at Finite Concentration," Journal of Colloid and Interface Science 73(1), 130-135 (1980).

4. M. Othman, A. Aschi, and A. Gharbi, "Polyacrylic acids-bovine serum albumin complexation: Structure and dynamics," Materials Science and Engineering C. 58, 316-323 (2016).

5. V. V. Gibizova, I. A. Sergeeva, G. P. Petrova, A. V. Priezzhev, and N. G. Khlebtsov, "Interaction of Albumin and gamma-Globulin Molecules with Gold Nanoparticles in Water Solutions," Moscow University physics bulletin. 66(5), 449-452 (2011) [in Russian].

6. http://www.photocor.ru/.

7. H. Z. Cummins, and E. R. Pike, Photon Correlation and Light Beating Spectroscopy, Springer Science+Business Media, New York (1974).

8. A. Einstein, Collected Papers, Nauka, Moscow (1966) [in Russian].

9. V. N. Tsvetkov, B. E. Eskin, and S. Y. Frenkel, Structure of Macromolecules in Solutions, Nauka, Moscow (1964) [in Russian].

\section{Introduction}

Proteins are an important component of a living organism executing different functions. The methods of dynamic and static light scattering are informative methods of studying the behaviour of protein molecules in solutions, using which one can determine the molecular mass, the coefficient of intermolecular interaction, the coefficient of translation diffusion. From the latter, using the Stokes-Einstein equation, one can estimate the hydrodynamic radius of the scattering particles. 
The analysis of the effect of different substances on the protein molecules allows the study of the processes that take place in a living organism. Under the development of different pathological changes in the organism, the parameters of protein molecules (total surface charge, intermolecular interaction coefficient, translation diffusion coefficient) can essentially vary.

Iron is one of widespread chemical elements taking part in the vital activity of living organisms. Iron is involved in blood formation, breathing, immune biological processes, and enters into the composition of more than a hundred enzymes.

Iron is a component of haemoglobin that executes the function of binding and delivery of oxygen to the cells of human organism. Therefore, iron is necessary for the synthesis and functioning of haemoglobin.

Iron is present in the human organism mainly as a component of a few biologically active compounds (haemoglobin, myoglobin, iron-containing enzymes) that execute four basic functions:

1. transport of electrons (cytochromes);

2. transport and deposition of oxygen (haemoglobin, myoglobin);

3. participation in the formation of active centres of redox enzymes;

4. transport and deposition of iron (transferrin, ferritin).

The concentration of iron in human blood considerably varies. It has a daily rhythm and the variations of its level in the blood serum of a healthy human during 24 hours can be as large as $~ 100 \%$.

Some diseases cause the change of iron content in the blood serum; thus, the decrease of the iron content is observed in the case of iron-deficit anaemia, the anomaly of iron absorption, and the increased level of iron is observed in the case of hemochromatosis [1].

In this connection, it is interesting to study the molecular dynamic processes that occur in the solutions of the main proteins of blood serum, comprising the iron ions.

In our study as an iron salt, we used Iron(III) chloride (this preparation is also intensely used in water purification).

Most substances enter the organism via the gastrointestinal tract and then appear in the blood circulation system. Albumin, the main protein of blood serum, is a globular protein, the main role of which in the organism is the transport of substances. In this connection, it is important to study the mechanism of interaction between the ferric chloride II and the albumin.

In the literature, there are practically no papers on the interaction of iron salts with serum proteins. However, there are a few papers, e.g., [2], in which the interaction of bovine serum albumin (BSA) with iron nanoparticles having the size of about $5 \mathrm{~nm}$ was studied. It was found that the iron nanoparticles with positive charges combine with the -COO-groups of the protein. They interact by means of electrostatic forces, thus forming aggregates of larger particles. In the paper the spectra of light scattered by the iron nanoparticles and by the mixture of iron nanoparticles and BSA were obtained. These spectra show that the intensity of light scattered by the iron nanoparticles is very small. However, when the nanoparticles are mixed with BSA the intensity of the scattered light strongly increases in the range of wavelengths $300-600 \mathrm{~nm}$ and reaches its maximum at $451 \mathrm{~nm}$ even for small concentrations of BSA (about $0.1 \mu \mathrm{g} / \mathrm{ml}$ ).

In other papers the aqueous solutions of BSA were studied [3, 4]. The Americal research team from Ohio determined the BSA translation diffusion coefficients in the concentration $0-200 \mathrm{mg} / \mathrm{ml}$ by means of laser light quasielastic scattering. The protein diffusion was studied in the systems having $\mathrm{pH}$ that was equal to 7.4 [3]. In this paper it was found that under the increase of BSA concentration the translation diffusion coefficient decreased insignificantly.

The research team from Tunisia University [4] studied the change of protein conformation depending on $\mathrm{pH}$. In the paper they studied the behaviour of the hydrodynamic radius of the particles affected by $\mathrm{pH}$, the scattering angle being $\theta=173^{\circ}$. It was shown that the hydrodynamic radius of the protein is constant in a wide range of $\mathrm{pH}$ values $\left(\mathrm{R}_{\mathrm{h}}\right.$ varied from 38 to $44 \AA$ ). These values are close to the results of earlier studies [5]. The increase of the protein molecule size at $\mathrm{pH}<3$ is an evidence of BSA denaturation [4].

\section{Experimental results}

All studies were carried out using the Photocor Complex setup [6], the laser wavelength being $647 \mathrm{~nm}$, and the power being $25 \mathrm{~mW}$.

\section{Preparation of solutions}

In the present work, we used the BSA (Sigma) and the $30 \%$ aqueous solution of iron(III) chloride (Panreac) as an iron-containing component.

As a solvent, we used the water for injections (OAO "Biokhimik", $\mathrm{pH}$ 7.0). The measurements were carried out at the temperature $20^{\circ} \mathrm{C}$.

For experiments it was necessary to prepare three solutions, with the normal (corresponding to a healthy organism) content of iron $(1.12 \mathrm{mg} / \mathrm{l})$, the increased $(3.35 \mathrm{mg} / \mathrm{l})$, and the decreased $(0.28 \mathrm{mg} / \mathrm{l})$ content, respectively. To prepare the first solution we used $10 \mu \mathrm{l}$ of $30 \%$ iron(III) chloride, which were then dissolved in $5 \mathrm{ml}$ of water. In the obtained solution (1) the mass of iron(III) chloride amounted to $13.4 \mathrm{mg}$. Form the new solution (1) we took $21 \mu \mathrm{l}$ and added to $5 \mathrm{ml}$ of water. As a result, we obtained the solution with the normal concentration of iron. For the solutions with increased and decreased concentration, we took $62 \mu \mathrm{l}$ and $5 \mu \mathrm{l}$ of the obtained aqueous solution (1) of the Iron(III) chloride, respectively, and added to $5 \mathrm{ml}$ of water. Then to each of these samples we added $20 \mu \mathrm{l}$ of BSA solution having the concentration $50 \mathrm{mg} / \mathrm{ml}$. 


\section{Experimental results obtained using the dynamic light scattering}

In the course of the experiment, we fixed the concentration of the ferric chloride and varied the BSA concentration. Basing on the results obtained using the dynamic light scattering method [7] the hydrodynamic radius $\left(R_{h}\right)$ and the translation diffusion coefficient $\left(D_{t}\right)$ were plotted versus the BSA concentration.
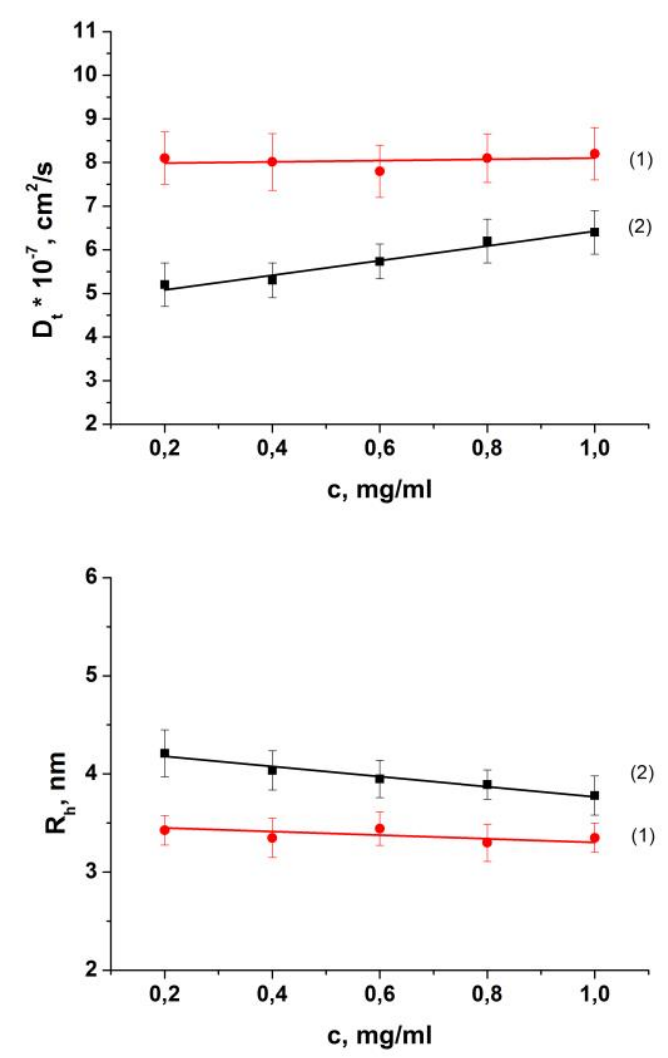

Fig. 1 Concentration dependence of the translation diffusion coefficient $\left(\mathrm{D}_{\mathrm{t}}\right)$ (top), and the hydrodynamic radius $\left(\mathrm{R}_{\mathrm{h}}\right)$ (bottom) for pure BSA (1) and for the BSA with the addition of aqueous solution of Iron(III) chloride with the content of $\mathrm{Fe}^{3+}$ equal to $0.28 \mathrm{mg} / \mathrm{l}$ (2).

The first series of experiments was carried out with the BSA solutions and with those in the presence of iron(III) chloride $(\mathrm{c}(\mathrm{Fe} 3+)=0.28 \quad \mathrm{mg} / \mathrm{l})$, which corresponds to the decreased content of iron in human blood (Fig. 1).
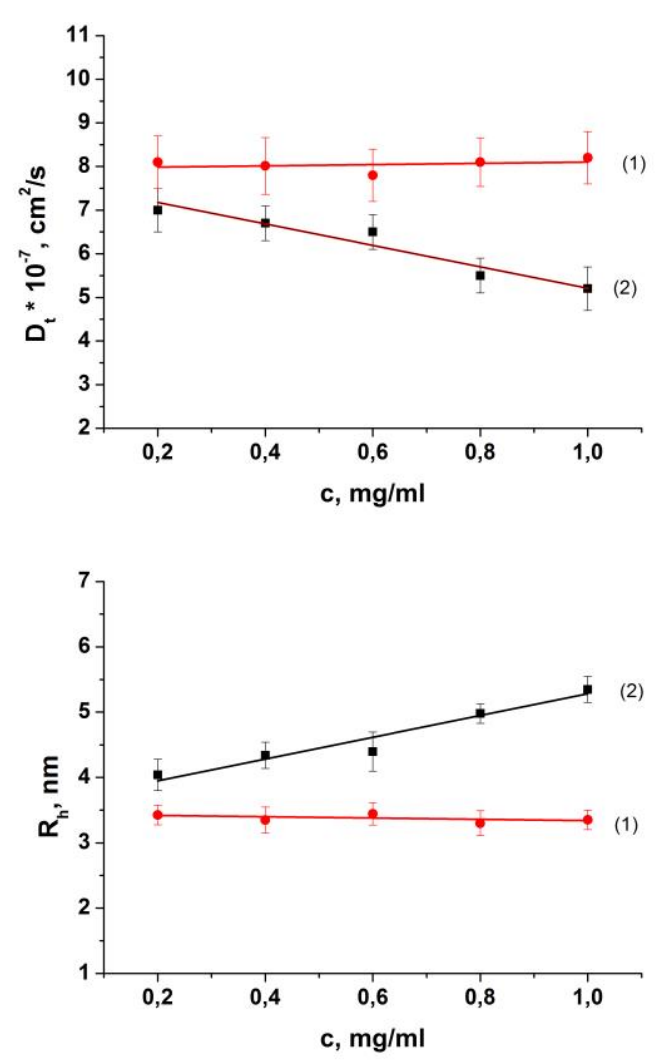

Fig. 2 Concentration dependence of the translation diffusion coefficient $\left(\mathrm{D}_{\mathrm{t}}\right)$ (top) and hydrodynamic radius (bottom) ) for pure BSA solution (1) and for the BSA with the addition of aqueous solution of iron(III) chloride with the content of $\mathrm{Fe}^{3+}$ equal to $1.12 \mathrm{mg} / \mathrm{l}$ (2).

Then we carried out a series of experiments with the solutions, containing the normal concentration of iron $\left(\mathrm{c}\left(\mathrm{Fe}^{3+}\right)=1.12 \mathrm{mg} / \mathrm{l}\right)$ (Fig. 2).

The last series of experiments was carried out with the increased concentration $\left(\mathrm{c}\left(\mathrm{Fe}^{3+}\right)=3.35 \mathrm{mg} / \mathrm{l}\right)$.

\section{Experimental results obtained by means of the static light scattering method}

Using the static light scattering [8] we determined the coefficient of intermolecular interaction $\boldsymbol{B}$ for BSA solutions with the addition of iron(III) chloride (Fig. 4) and the molecular mass of the scattering particles $\boldsymbol{M}$ (Fig. 5). 

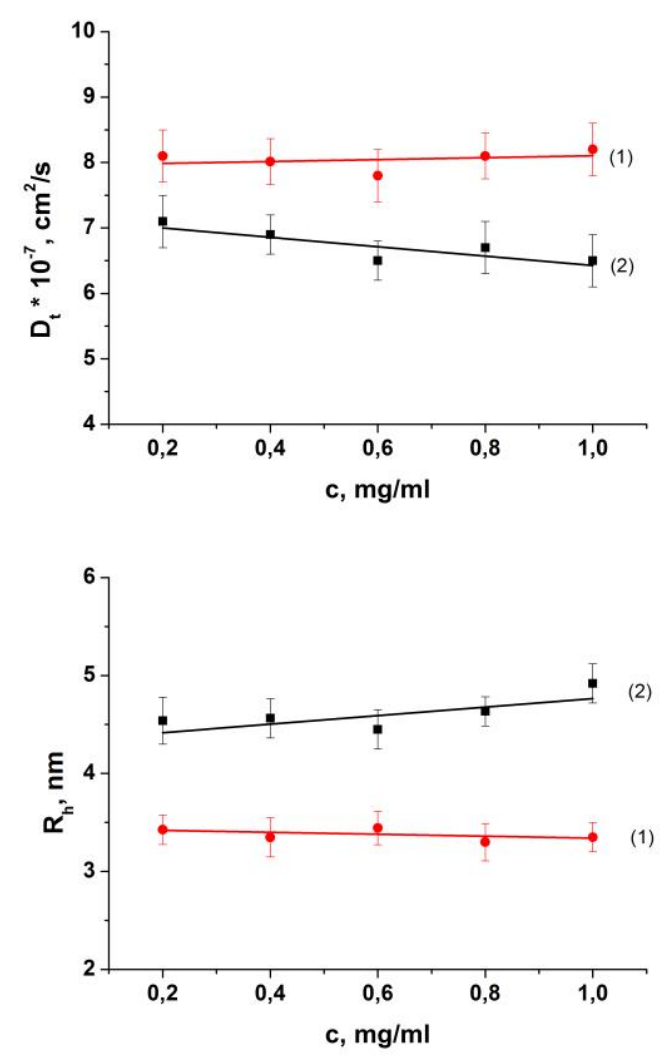

Fig. 3 Concentration dependence of the translation diffusion coefficient (Dt) (top) and hydrodynamic radius (bottom)) for pure BSA solution (1) and for the BSA with the addition of aqueous solution of Iron(III) chloride with the content of $\mathrm{Fe} 3+$ equal to $3.35 \mathrm{mg} / \mathrm{l}$ (2).

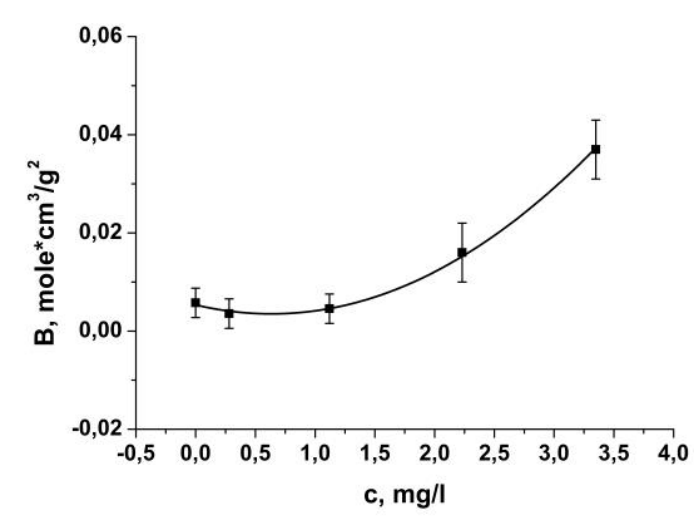

Fig. 4 Dependence of the coefficient of intermolecular interaction upon the concentration of $\mathrm{Fe}^{3+}$.

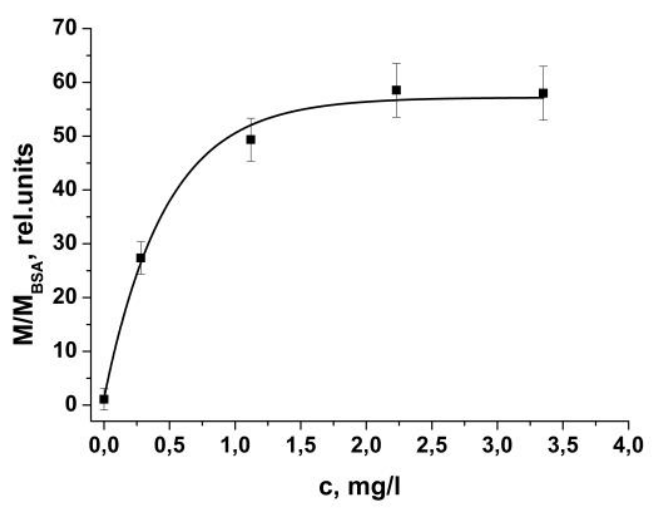

Fig. 5 Dependence of the ratio of the molecular mass of the scattering particles to that of BSA upon the concentration of $\mathrm{Fe}^{3+}$.

\section{Discussion}

The obtained values of the hydrodynamic radius of particles in the solution exceed the size of BSA molecules in the pure solution by less than $60 \%$. This fact can mean that no protein aggregates are formed.

The growth of molecular size can be explained by partial absorption of ions that appear due to the interaction between the iron(III) chloride with water on the BSA molecules. The model of this process is presented in Fig. 6.

At the same time an insignificant decrease of the translation diffusion coefficient and increase of the intermolecular interaction coefficient occur, caused by the addition of $\mathrm{FeCl}_{3}$.

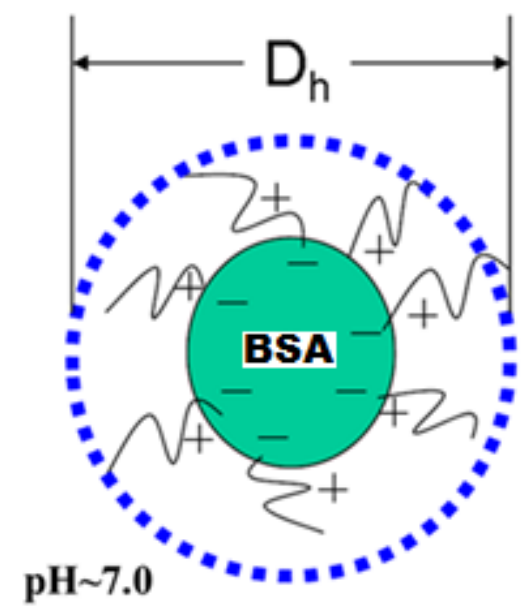

Fig. 6 Model of partial adsorption of iron ions on the BSA protein. 
Table 1 The ratio of the scattering particle mass to that of the pure albumin depending on the ionic strength of the solution

\begin{tabular}{|l|l|l|l|l|l|}
\hline $\mathrm{I}, \mathrm{mmole} / \mathrm{l}$ & 0 & 0.025 & 0.1 & 0.2 & 0.3 \\
\hline $\begin{array}{l}\mathrm{M} / \mathrm{M}_{\mathrm{BSA}}, \\
\text { rel. units }\end{array}$ & 1.09 & 27.03 & 49.3 & 58.5 & 58 \\
\hline
\end{tabular}

The results obtained in the course of measurements for the molecular mass of scattering particles in the BSA solution with the addition of Iron(III) chloride (Table 1) were compared with the mass of the particles in the pure solution of Iron(III) chloride (Table 2). According to the Rayleigh-Debye theory, one can find the mass of the scattering particles using the expression

$$
\frac{c H K}{R_{90}}=\frac{1}{M}+2 B c+\cdots
$$

where $c$ is the concentration of the solution, $H$ is the optical constant of the solution, $K$ is the Cabanne factor, $R_{90}$ is the reduced intensity for the angle $90^{\circ}, B$ is the second virial coefficient, characterising the degree of the solution imperfection and allowing for pair intermolecular interactions in the solution [9]. The obtained dependence of $R_{90}$ on $c$ does not allow one to determine the mass using the measurement for one concentration. It is necessary to perform measurements for a few values of $\mathrm{c}$ and to extrapolate the results to $c=0$, which yields the following result:

$$
M=\left(\frac{c H K}{R_{90}}\right)_{c \rightarrow 0}^{-1}
$$

Table 2 The obtained values of the mass of scattering particles and the intermolecular interaction coefficient

\begin{tabular}{|l|l|l|}
\hline & Value & Error \\
\hline $\mathrm{M}^{*} 10^{6}, \mathrm{~g} / \mathrm{mole}$ & 3.25 & 0.24 \\
\hline $\mathrm{B}, \mathrm{mole}^{*} \mathrm{~cm}^{3} \mathrm{~g}^{2}$ & 0.601 & 0.012 \\
\hline
\end{tabular}

The calculated value of the mass of particles in the Iron(III) chloride solution provides a thorough explanation the sharp increase of the scattering particles under the addition of protein BSA molecules to the solution. The heavy ions that apparently arise in the interaction of the complex compounds of iron that have the radius by 50 times smaller than that of the DSA. The existence of the ion adsorption process i.e., the binding of these heavy complexes to protein molecules becomes theoretically possible, which considerably increases the total mass of the forming particles leaving their radius of these complexes practically unchanged.

\section{Conclusion}

The study of the behaviour of protein molecules in the albumin solutions, containing the Iron(III) ions is carried out and the features of molecular mobility of albumin molecules in the pure aqueous solution and with the addition of Iron(III) chloride are investigated.

The obtained results allowed the following conclusions:

1. The study of the dynamic parameters of serum albumin under the action of iron(III) ions using the method of photons correlation has shown that the hydrodynamic radius of the scattering particles insignificantly increases due to the addition of iron(III) chloride (by less than $60 \%$ ).

2. We have found a significant increase of the mass of scattering particles in BSA solutions under the addition of iron(III) chloride, which can be caused by the formation of heavy ions in the solution of iron(III) chloride due to the interaction of $\mathrm{FeCl}_{3}$ with water.

3. The process of partial adsorption of heavy ion complexes on the negatively charged BSA molecules was observed.

4. It was shown that with the increase of the concentration of $\left(\mathrm{Fe}^{3+}\right)$ the molecular mass of scattering particles becomes constant. The observed effect can be a consequence of the Langmuir saturation process in the course of the monolayer formation.

It can be summarised that the influence of Iron(III) on the molecular mobility of serum albumin is insignificant.

\section{Acknowledgments}

The study was supported by the Russian Foundation for Basic Research, project No. 16-32-00847 mol_a. 